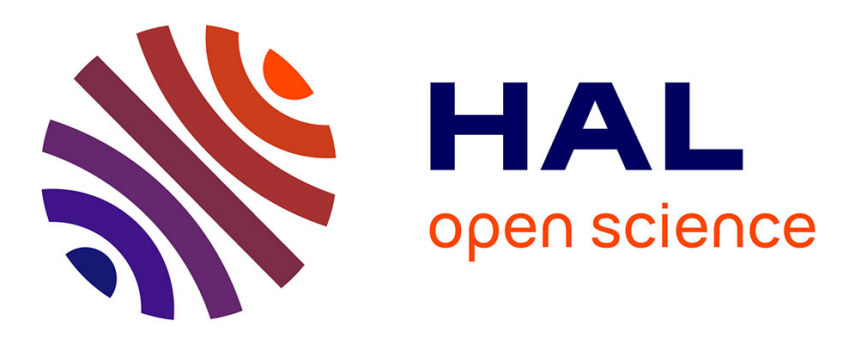

\title{
Energy balance properties of steels subjected to high cycle fatigue
}

André Chrysochoos, Antoine Blanche, Bruno Berthel, Bertrand Wattrisse

\section{To cite this version:}

André Chrysochoos, Antoine Blanche, Bruno Berthel, Bertrand Wattrisse. Energy balance properties of steels subjected to high cycle fatigue. SEM 2011, 2011, United States. hal-00832724

\section{HAL Id: hal-00832724 \\ https://hal.science/hal-00832724}

Submitted on 11 Jun 2013

HAL is a multi-disciplinary open access archive for the deposit and dissemination of scientific research documents, whether they are published or not. The documents may come from teaching and research institutions in France or abroad, or from public or private research centers.
L'archive ouverte pluridisciplinaire HAL, est destinée au dépôt et à la diffusion de documents scientifiques de niveau recherche, publiés ou non, émanant des établissements d'enseignement et de recherche français ou étrangers, des laboratoires publics ou privés. 


\title{
Energy balance properties of steels subjected to high cycle fatigue
}

\author{
A. Chrysochoos ${ }^{1}$, A. Blanche ${ }^{1}$, B. Berthel ${ }^{2}$, B. Wattrisse ${ }^{1}$, \\ (1) LMGC, UMR CNRS 5508 Montpellier University, CC 048, Place E. Bataillon, 34095 \\ Montpellier, France \\ (2) LTDS, UMR CNRS 5513, École Centrale de Lyon, 36 Avenue Guy de Collongue, 69134 \\ Ecully, France
}

\begin{abstract}
The paper presents an experimental protocol developed to locally estimate the different terms of the energy balance associated with the high cycle fatigue (HCF) of steels. Namely, the deformation and dissipated energy are respectively derived from displacement and temperature fields obtained using digital image correlation (DIC) techniques and quantitative infrared thermography (QIRT). The combined processing of visible and infrared images shows the precocious, gradual and heterogeneous development of fatigue localization zones, It also shows the plastic character of dissipative heat sources (i.e. proportional to the loading frequencies) and the non negligible amounts of internal energy variations during a so-called elastic loading cycle. The progress of the fatigue dissipation is finally underlined constructing the drift of the mean dissipation per cycle for a given loading. The paper ends with discussing the consequences of such energy balance properties in terms of HCF modeling.
\end{abstract}

\section{Introduction}

Standard characterization of fatigue in materials requires time-consuming and statistical processing of the numerous results provided by expensive mechanical tests. Over the last two decades, alternative experimental approaches have been developed to rapidly produce reliable fatigue characteristics. These new approaches include thermal methods based on the analysis of self-heating during stepwise loading fatigue tests [1-2]. Although realistic estimates of fatigue characteristics have sometimes been obtained, the thermal approach often leads to questionable results [3]. The direct use of temperature as a fatigue indicator may not always be the most relevant approach since the temperature variation is not intrinsic to the material behavior. Energy approaches were consequently proposed [4-6 (Mabru-Boulanger-Berthel].

During high cycle fatigue, a first part of the evolved heat comes from irreversible manifestations of micro-structural defects (e.g. intra-granular slip bands), while another part comes from thermoelastic coupling sources linked to the reversible thermal expansion of the crystalline network. A combined image processing was then developed to obtain 2D patterns of energy balances. Speckle image correlation techniques, involving a charge-coupled device (CCD) camera [7], were used to assess surface displacement fields. In parallel, thermal data were provided by an infrared focal plane array (IRFPA) camera [8]. Heat sources were estimated on the basis of partial derivative operators present in a local form of the heat diffusion equation by using a set of approximation functions that locally fits the displacement and temperature field and takes the spectral properties of the sought sources into account.

The present paper shows several important properties associated with the fatigue of a dual-phase steel often used in mechanical industries. This paper is composed as follows: the different terms of the energy balance associated with high cycle fatigue of steel are first recalled. A brief presentation of the experimental arrangement is then given, the main part of the paper being devoted to the presentation of the energy balance properties. In particular, the precocious, gradual and heterogeneous development of the fatigue localization is shown. Then the "plastic" character of dissipation (i.e. proportional to the loading frequency $f_{\mathrm{L}}$ ) is underlined. Experiments also reveal non negligible amounts of internal energy variations (stored energy) during a so-called elastic loading cycle 
and show the evolution of the mean dissipation per cycle during blocks of cycles at constant stress range $\Delta \sigma$ for a given $f_{\mathrm{L}}$ and a given stress ratio $R_{\sigma}$. The paper ends with discussing the consequences of such energy properties in terms of HCF modeling.

\section{Theoretical background}

a. Heat equation: concepts and results of the thermodynamics of irreversible processes must be used to define the different heat sources induced by the HCF processes. In this paper the thermodynamics with internal state variables will be used, whereby the equilibrium state of each volume material element is characterized by a set of $n$ state variables. The chosen state variables are the absolute temperature $T$, the small strain tensor $\varepsilon$, and the $n$ 2 scalar components $\left(\alpha_{1}, \ldots, \alpha_{n-2}\right)$ of the vector $\alpha$ of so-called "internal" variables that model the micro-structural state of the material. By construction, the thermodynamic potential is the Helmholtz free energy $\psi$. Combining the first and second principles of thermodynamics leads to the local heat diffusion equation:

$$
\rho C \dot{T}-\operatorname{div}(k \operatorname{grad} T)=\underbrace{\sigma: \dot{\varepsilon}-\rho \psi_{, \varepsilon}: \dot{\varepsilon}-\rho \psi_{, \alpha} \cdot \dot{\alpha}}_{d_{1}}+\underbrace{\rho T \psi_{, \varepsilon T}: \dot{\varepsilon}+\rho T \psi_{, \alpha} \cdot \dot{\alpha}}_{S_{\mathrm{thc}}}+r_{\mathrm{e}},
$$

where $\rho$ denotes the mass density, $C$ the specific heat, $k$ the heat conduction tensor, $\sigma$ the Cauchy stress tensor and $\dot{\varepsilon}$ strain rate tensor. The left-hand side consists of a differential operator applied to the temperature, while the right-hand side gathers the various types of heat sources: the intrinsic dissipation $d_{1}$, the thermo-mechanical coupling sources $s_{\mathrm{thc}}$, and the possible external heat supply $r_{\mathrm{e}}$ (e.g. radiation exchanges). The intrinsic dissipation characterizes the material degradation accompanying the irreversible transformation of the microstructure, while the thermo-mechanical heat sources translates the thermo-sensitivity of the matter, indicating the mechanical and thermal states are closely coupled. When the couplings are only due to thermo-elasticity, the coupling sources are limited to the famous Lord Kelvin's term

$$
s_{\text {the }}=\rho T \psi_{, \varepsilon T}: \dot{\varepsilon}
$$

Up to now, the following strong hypotheses have been put forward to compute the heat sources:

- the mass density and the specific heat are material constants, independent of the thermodynamic state.

- the heat conduction tensor remains constant and isotropic during the test $\left(k_{\mathrm{ij}}=k \cdot \delta_{\mathrm{ij}}\right)$.

- the external heat supply $r_{\mathrm{e}}$ due to heat exchange by radiation is time-independent, so the equilibrium temperature field $T_{0}$ verifies $-\Delta T_{0}=r_{\mathrm{e}}$. It is then convenient to consider the temperature variation $\theta$ defined by $\theta=T-T_{0}$.

Taking into account these last hypotheses, the heat equation can be rewritten in the following compact form:

$$
\rho C \dot{\theta}-k \Delta \theta=w_{h},
$$

where $\dot{\theta}$ is the particular time derivative of the temperature variation, $\Delta$ the 3D Laplacian operator, $w_{h}$ the overall heat source, $w_{\mathrm{h}}$ being the heat locally evolved and where the non standard dot (.) ${ }^{\circ}$ specifies that the energy rate is path dependent (i.e. such energy is not a state function).

These hypotheses are reasonable in many situations and in particular at the beginning of HCF processes. However, they become inadequate when a strong anisotropy pre-exists or develops during the loading, whether a strong strain and/or damage localization occurs or whether the thermo-mechanical loading leads to dynamic instabilities.

b. Energy balance: let a load-unload cycle be with $A=\left(T_{A}, \varepsilon_{A}, \alpha_{A}\right)$ and $B=\left(T_{B}, \varepsilon_{B}, \alpha_{B}\right)$ denoting the thermomechanical states of the material at the extremities of the process. Let us draw up the energy balance corresponding to the 3 following situations:

(case 1) $A \neq B$ : this general situation can be illustrated by the schematic stress-strain diagram proposed in Fig. 1. (case 2) $A \neq B$ and $\varepsilon_{A}=\varepsilon_{B}$ : a mechanical cycle is then associated with the load-unload test.

The stress-strain diagram shows a hysteresis loop.

(case 3) $A=B$ : the mechanical cycle is now a thermodynamic cycle.

For quasi-static process and under the small strain hypothesis, the rate of deformation energy is classically defined by: 


$$
w_{\text {def }}=\sigma: \dot{\varepsilon}
$$

Following Eq.(1), the intrinsic dissipation $d_{1}$ is then the difference between the deformation energy rate $w_{\text {def }}^{\cdot}$, and the sum of the elastic $w_{\mathrm{e}}^{\cdot}$ and stored $w_{\mathrm{s}}^{\cdot}$ energy rates:

$$
\begin{aligned}
& d_{1}=w_{\mathrm{d}}^{\cdot}=w_{\text {def }}^{\cdot}-w_{\mathrm{e}}^{\cdot}-w_{\mathrm{s}}^{\cdot}, \\
& w_{\mathrm{e}}^{\cdot}+w_{\mathrm{s}}^{\cdot}=\rho \psi_{, \varepsilon}: \dot{\varepsilon}+\rho \psi_{, \alpha} \cdot \dot{\alpha} .
\end{aligned}
$$

Both principles of thermodynamics give an alternative expression of the volume deformation energy associated with the load-unload test:

$$
w_{\text {def }}=\int_{t_{A}}^{t_{B}} d_{1} \mathrm{~d} t+\int_{t_{A}}^{t_{B}}\left(w_{\mathrm{e}}^{\cdot}+w_{\mathrm{s}}^{\cdot}\right) \mathrm{d} t=\int_{t_{A}}^{t_{B}} d_{1} \mathrm{~d} t+\int_{t_{A}}^{t_{B}}\left(\rho \dot{e}-\rho C \dot{\theta}+w_{\text {the }}^{\cdot}+w_{\text {thc }}^{\cdot}\right) \mathrm{d} t
$$

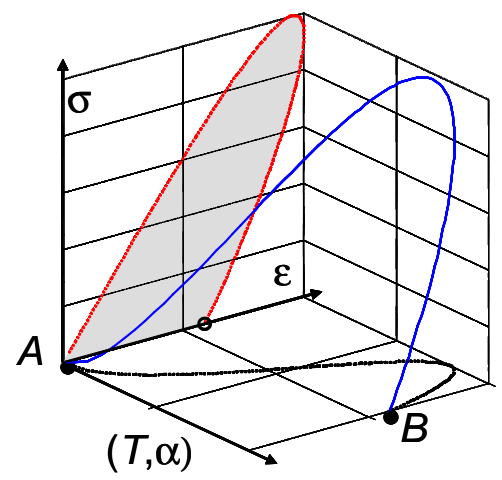

Fig. 1: Schematic stress-strain diagram for a load-unload test; $t_{\mathrm{B}}-t_{\mathrm{A}}$ is the cycle duration

Equation (7) shows that:

(case 1) In the general case, the balance of deformation energy during a load unload test involves, energy dissipation, internal energy variations, variation of heat stored in the material, coupling heat sources.

(case 2) For a mechanical cycle, the deformation energy then corresponds to the energy $A_{\mathrm{h}}$ of the area of the hysteresis loop.

(case 3) For a thermodynamic cycle, this hysteresis loop is then only due to dissipation and couplings as soon as the volume heat capacity $\rho C$ is supposed to be constant.

$$
w_{\text {def }}=A_{\mathrm{h}}=\int_{t_{A}}^{t_{B}} d_{1} \mathrm{~d} t+\int_{t_{A}}^{t_{B}}\left(w_{\text {the }}+w_{\text {thc }}\right) \mathrm{d} t
$$

The energy balance form then gives the restricted conditions for which the dissipated energy can be estimated by computing the hysteresis area of a uniaxial load-unload cycle. This underlines the necessity of analyzing the thermal effects to verify if a mechanical cycle is also a thermodynamic cycle, and checking the relative importance of coupling effects. It is worth noting that in the case of HCF of DP 600 steel, the thermoelastic energy remains negligible over a complete cycle. Moreover, after some cycles, the temperature becomes periodic and the mean temperature variation stabilizes. In such a case, the deformation energy over a loading cycle is made of dissipated energy and internal energy variation.

$$
w_{\mathrm{def}}=\int_{t A}^{t B} d_{1} \mathrm{~d} t+\int_{t A}^{t B} \rho \dot{e} \mathrm{~d} t=w_{\mathrm{d}}+[\rho e]_{A}^{B}
$$

If the deformation energy is equal to the dissipated one, the mechanical cycle is a thermodynamic cycle (case 3) and there is no overall evolution of the microstructure over a cycle. A mechanical illustration of this situation is the perfect elastic-plastic shakedown. If the deformation energy is greater than the dissipated one (neglecting coupling heats over a cycle), the material stores some internal energy (case 2). This stored energy is used by the material to modify its microstructure. If the deformation energy becomes less than the dissipated energy, a part of 
the stored energy is then transformed into dissipated energy which means that the material is no longer able to adapt its microstructure.

Note that dividing volume energy (resp. energy rate) by volume heat capacity $\rho \mathrm{C}$ of the material gives volume energy (resp. energy rate) expressed in ${ }^{\circ} \mathrm{C}$ (resp. in ${ }^{\circ}$. $s^{-1}$ ). This operation is convenient because it associates an equivalent heating in adiabatic conditions and then gives order of magnitudes of this energy (resp. energy rate) in unit easy to grasp.

\section{Experimental arrangement}

The experimental set-up, designed to derive local energy balances, involves a MTS hydraulic testing machine (frame: $100 \mathrm{kN}$, load cell: $25 \mathrm{kN}$ ), a Camelia $8 \mathrm{M}$ high resolution CCD camera and a Cedip Titanium infrared camera. The optical axis of both cameras was set perpendicularly to the frame of the testing machine, and remained fixed during the test. A simultaneous record of infrared and visible images was performed on each side of the sample surface, and thin flat samples were therefore used.

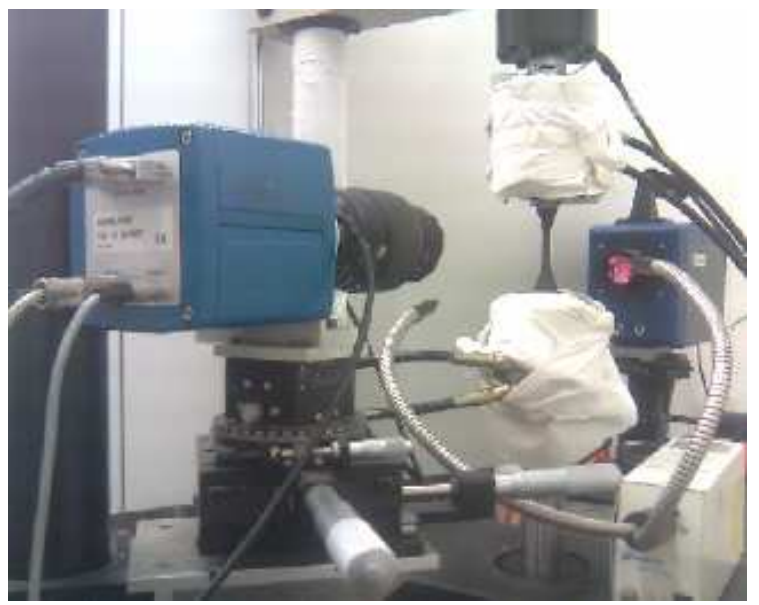

Fig. 2: Experimental arrangement. (Front): CCD camera and cold light sources, (back): IRFPA camera. The grips and columns of the testing machine are roughly wrapped to avoid parasitic reflection

The surface in front of the CCD camera was speckled with white paint on a black background to get an optimized contrast of local optical signature, while the surface emissivity of the sample gauge part placed in front of the IR camera was increased and homogenized using a mat black paint. Typically the size of the analyzed zone was about $10 \times 10 \mathrm{~mm}^{2}$ for space resolution of about $0.1 \mathrm{~mm}$. The conventional strain rates were less than $10^{-2} \mathrm{~s}^{-1}$. This order of magnitude of strain rate enables the quasi-staticity of processes to be ensured and induces significant (thermal) signal-to-noise ratios.

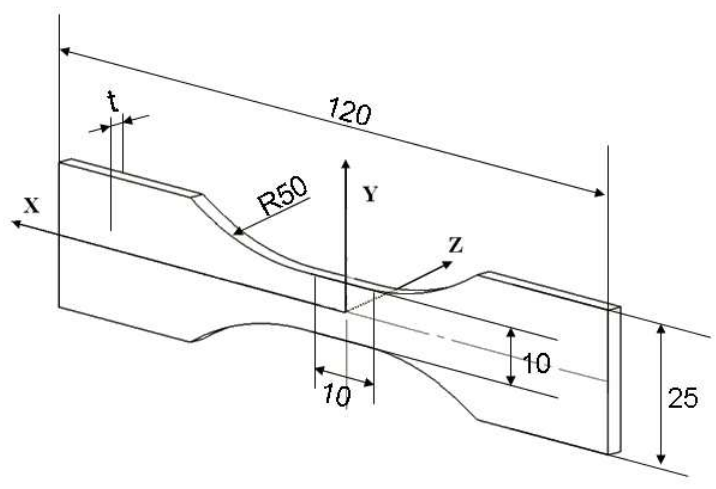

Fig.3: Shape of the specimen (unit: $\mathrm{mm}$ ) and frame of reference 
Each camera was controlled by a separate computer although a specific electronic device was designed to synchronize the frame grabbing of the two cameras. Using this device, the synchronization error between the two cameras is estimated to be less than $0.05 \mathrm{~ms}$.

Reference speckle and infrared images were mapped by determining the affine transformation coefficients (rigid body movement and homothetic transformation) of the shape of a calibrated target. The sample shape corresponded to a standard dog-bone specimen. The main material constants of the DP600 steel are grouped in Table 1.

\begin{tabular}{cccc}
\hline$\lambda_{\text {th }}\left(10^{-6} \cdot{ }^{-} \mathrm{C}^{-1}\right)$ & $\rho\left(\mathrm{kg} \cdot \mathrm{m}^{-3}\right)$ & $C\left(\mathrm{~J} \cdot \mathrm{kg}^{-1} \cdot{ }^{\mathrm{C}}{ }^{-1}\right)$ & $k\left(\mathrm{~W} \cdot \mathrm{m}^{-1} \cdot \mathrm{C}^{-1}\right)$ \\
\hline $10-11$ & 7800 & 460 & 64 \\
\hline
\end{tabular}

Table 1: Thermophysical properties of DP600

For each acquisition time the thermal data given by the IR camera (measured in the current, Eulerian configuration) were linearly interpolated in space and time using the positions of the deformed configuration given by the DIC computation. This operation allowed the material surface element (mse) associated with the DIC mesh to be tracked so that the extent of the volume heat rate induced by matter convection and associated with the particular time derivative of the temperature could subsequently be computed and checked. Readers interested in the many tricky metrology problems and in a detailed presentation of image processing can refer to [4-6].

An appropriate image processing method was developed to estimate separately fields of mean intrinsic dissipation per cycle $\bar{d}_{1}$ and fields of thermoelastic source amplitude $\Delta \mathrm{s}_{\text {the }}$. [Boulanger - Berthel].

The local fitting function $\theta^{\text {fit }}$ of the temperature charts is chosen as:

$$
\theta^{\mathrm{fit}}(x, y, \tau)=p_{1}(x, y) \tau+p_{2}(x, y)+p_{3}(x, y) \cos \left(2 \pi f_{L} \tau\right)+p_{4}(x, y) \sin \left(2 \pi f_{L} \tau\right)
$$

where the trigonometric time functions describe the periodic part of the thermoelastic effects while the linear time function takes transient effects due to heat losses, dissipative heating and possible drifts in the equilibrium temperature into account. Functions $p_{\mathrm{i}}(x, y), i=1, \ldots, 4$, are $2^{\text {nd }}$ order polynomials in $x$ and $y$. These polynomials enabled us to take into account the possible spatial heterogeneity of the source patterns [Berthel].

\section{Experimental results}

\section{a. Description of tests}

Tests involved loading blocks, each block being performed at constant $\Delta \sigma$ with $R_{\sigma}=-1$ and $f_{\mathrm{L}}=30 \mathrm{~Hz}$. Tests were made of stages (Fig. 4), each stage containing $S_{i}, i=1,2, \ldots$ :

- a series of 5 "mini" blocks of 3000 cycles per block with increasing $\Delta \sigma$ varying regularly from 175 up to $560 \mathrm{MPa}\left(m_{\mathrm{i}}\right)$

- a large block of 100000 cycles performed at $\Delta \sigma=580 \mathrm{MPa}\left(p_{\mathrm{i}}\right)$.

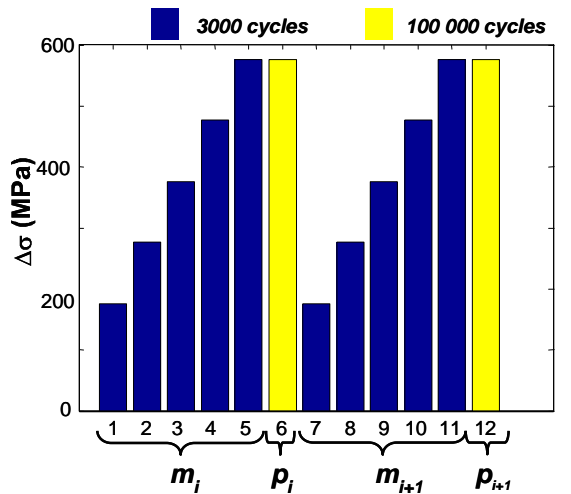

Fig.4: Basic sketch of the fatigue test 
Stages were repeated until a macro-crack occurs. The series of mini blocks was performed to construct the curve $\bar{d}_{1}$ vs. $\Delta \sigma$ at "constant" fatigue state, while the large blocks was defined, close to the fatigue limit, to speed up the fatigue progress. During the large blocks, camera shoots were regularly done to check the monotonous evolution of dissipative effects.

\section{b. Mechanical response}

To visualize the hysteretic mechanical response of the material during a loading cycle, and to improve the computation of the deformation energy corresponding to the hysteresis area of the stress-strain curve (Fig. 3), the CCD camera data acquisitions were performed at $f_{L}=5.10^{-3} \mathrm{~Hz}$ to increase the $f_{S} / f_{L}$ ratio, with $f_{S}$ denoting the frame rate of the camera $\left(f_{\mathrm{S}}=3 \mathrm{~Hz}\right)$.

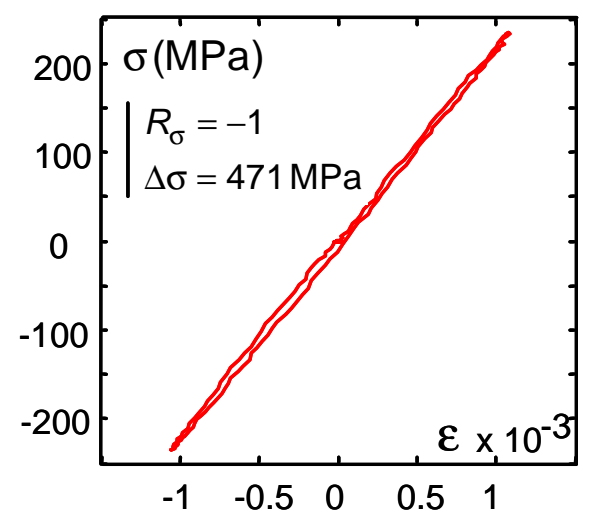

Fig.5: Mechanical hysteresis loop corresponding to case 2.

It is worth noting that the computation of the stress state fields was made assuming a plane stress state and an isochoric transformation. These hypotheses are naturally disputable, particularly at the end of the specimen's life where the triaxiality of the stress state is certainly coupled with the damage development. Note also that the complete passage from strain to stress field measurements has been already detailed in [JoMMS]. The relation used to compute the mean deformation energy rate over a cycle was then:

$$
w_{\text {def }}=f_{L} \int_{t_{A}}^{t_{A}+f_{L}^{-1}}\left(\sigma_{x x} \dot{\varepsilon}_{x x}+2 \sigma_{x y} \dot{\varepsilon}_{x y}+\sigma_{y y} \dot{\varepsilon}_{y y}\right) \mathrm{d} t
$$

\section{c. Thermoelastic response}

Until the onset of a crack, we observed quasi-uniform patterns of thermoelastic nature as expected for a simple tension-compression test. Besides, the experiments showed that $\Delta \mathrm{s}_{\text {the }}$ increased linearly with stress and the load frequency as predicted by a linear thermoelastic law [Boulanger]. An example of distribution of thermoelastic source amplitudes is given in Fig. 6 .

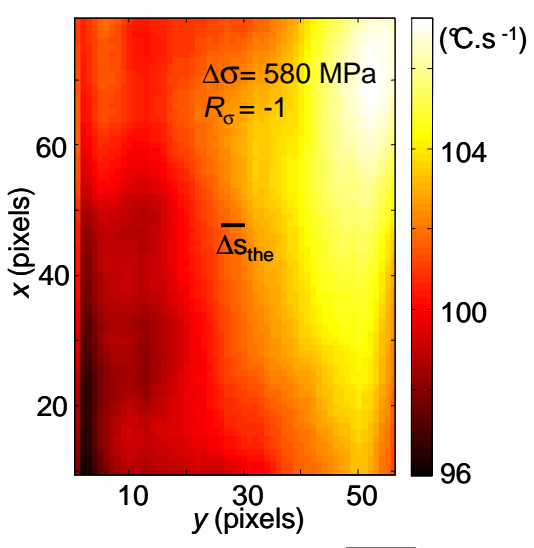

Figure 6: Mean 2D distribution of thermoelastic sources $\overline{\Delta s_{\text {the }}}(x, y)$ averaged over the $5^{\text {th }}$ loading block 
This 2D distribution is the mean thermoelastic source amplitudes averaged over the fifth loading block. The two main features to be noticed are the quite good homogeneity of the coupling source field and the order of magnitude of the thermoelastic effects. The mean value of $\overline{\Delta s_{\text {the }}}$ over the gage part of the specimen is around $101.9^{\mathrm{C}} \mathrm{C} . \mathrm{s}^{-1}$ and the standard deviation of about $2.4^{\mathrm{C}} \mathrm{C} . \mathrm{s}^{-1}$. Considering HCF of steel, this order of magnitude of heat source amplitude is typically a hundred to a thousand times greater than the dissipation intensities as shown hereafter (cf. Fig.7). This difference of order of magnitude of both types of sources complicates singularly the image processing. Indeed, the underlying dissipation sources, even those of low intensity, are of major importance to understand the fatigue mechanism development, and must be carefully extracted from the overall heat sources.

\section{d. Dissipation fields properties}

Contrary to thermoelastic source fields, heterogeneous distribution of dissipative sources were systematically observed (Fig. 7a). During a fatigue block, this pattern remains spatially constant whatever the set of loading parameters as long as fatigue effects are low and do not herald a crack inception [Berthel]. We associated the time independence of the heterogeneous distribution of dissipation with the low development of persistent slip bands (PSB) concentrated in few grains. We then interpreted the rapid evolution and the strong narrowing of highest dissipation zone as a sign of material damage leading to the crack inception. So, the experimental method enabled us to detect this crack localization several thousands of cycles before its onset.

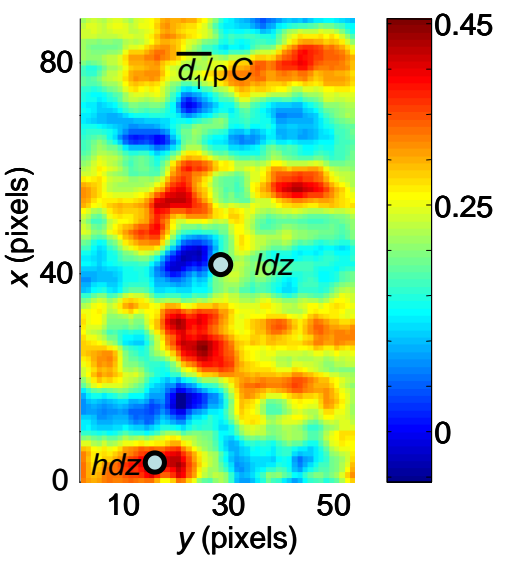

Figure 7: Example of heterogeneous distribution of dissipation averaged over a mini block: $t_{\mathrm{L}}=30 \mathrm{~Hz}$, $\Delta \sigma=500 \mathrm{MPa}, R_{\sigma}=-1$. The circles locate a low and high dissipation zone respectively.

Besides the calorimetric analysis showed that $\bar{d}_{1}$ linearly increases with the loading frequency at constant loading amplitude in every point of the sample gage part and despite the heterogeneous response of the material. Fig. $7 \mathrm{~b}$ shows the mean intrinsic dissipation per block $\bar{d}_{1}$ as a function of $\Delta \sigma$ and of $f_{L}$.
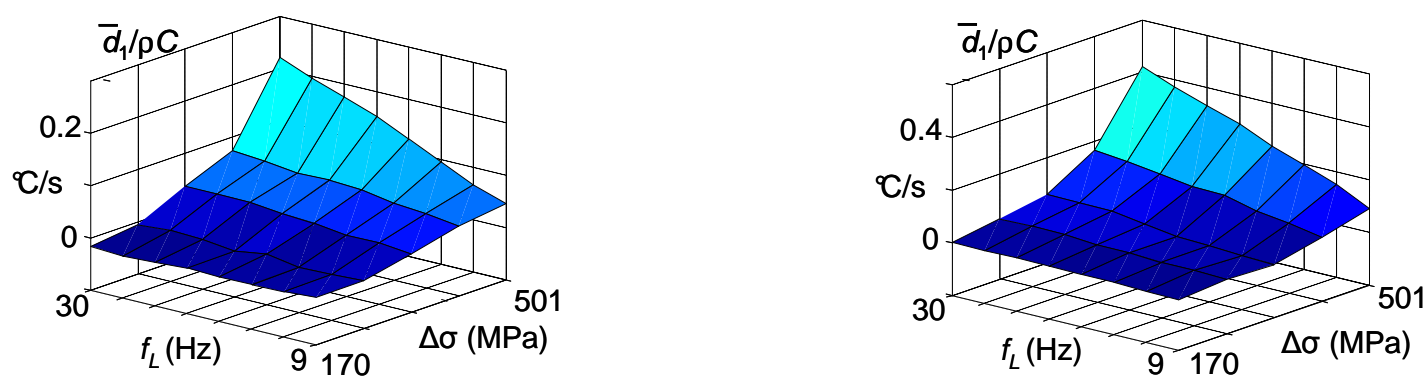

Figure 8: Evolution of the mean dissipation per (mini) block as a function of the stress range $\Delta \sigma$ and of the loading frequency $f_{\mathrm{L}}$. On the left: "low" dissipation zone, on the right: "high" dissipation zone.

Two zones were chosen (low/high dissipation zones) to show that whatever the zone, the dissipation surfaces are similar, which means that the microstructural mechanisms are probably the same if they do not have the same intensity. In particular, the linear evolution of the dissipation as a function of the loading frequency is compatible 
with the rate independent formalism of the plastic damageable materials. No viscous nor yield effects are here observable from a dissipative point of view.

Fig. 9 shows the drift of intrinsic dissipation along with the number of cycle for different stress ranges. The mean dissipation values were computed for the period corresponding to a mini block (i.e. 100 seconds) and averaged over the sample gage part. The curves associated with mini-block series are interpreted as the dissipative signature of the microstructural state at different stress range for an approximately constant fatigue state. This signature is associated with the number of active PSBs. These PSBs are randomly oriented within a few of grains and the greater the stress intensity, the larger the number of activated PSBs. During the large blocks, performed close to the fatigue limit $\left(\Delta \sigma_{L} \approx 540 \mathrm{MPa}\right.$ when $\left.R_{\sigma}=-1\right)$, the monotonous increase of dissipation during such blocks is the energy manifestation of the fatigue progress.

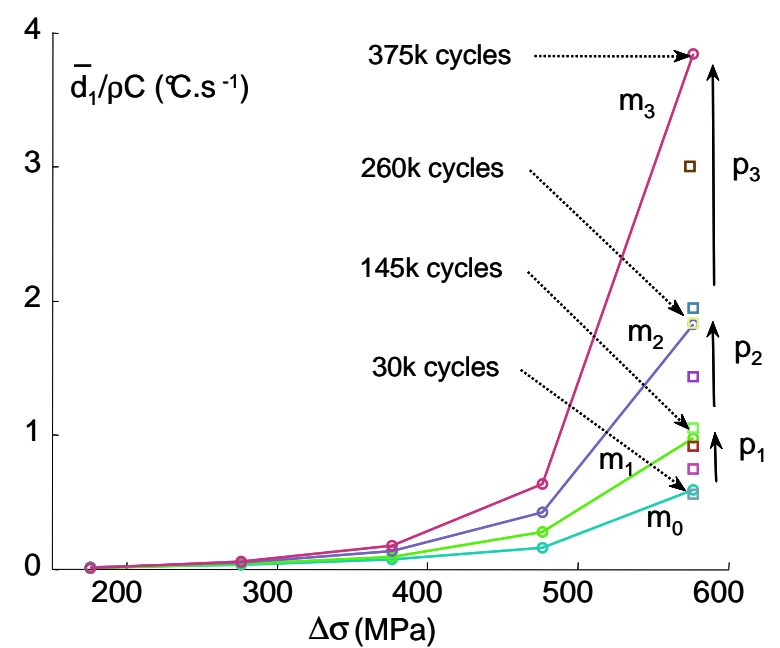

Figure 9: Drift of the mean dissipation.

\section{e. Discussion about the size of dissipation zones}

In Fig. 7, we observe that the order of magnitude of zones that dissipate preferentially is about 10 to 20 pixels corresponding to 1 to $2 \mathrm{~mm}$. This size may be naturally surprising if we refer to the size of PSBs which is about 1 $\mu \mathrm{m}$. To be convinced by such mesoscopic effects, we consider that dissipative heat sources are induced by active PSBs. The size of grains is chosen equal to $10 \mu \mathrm{M}$ and we suppose that only a few of grains have PSBs (see Fig. 10).

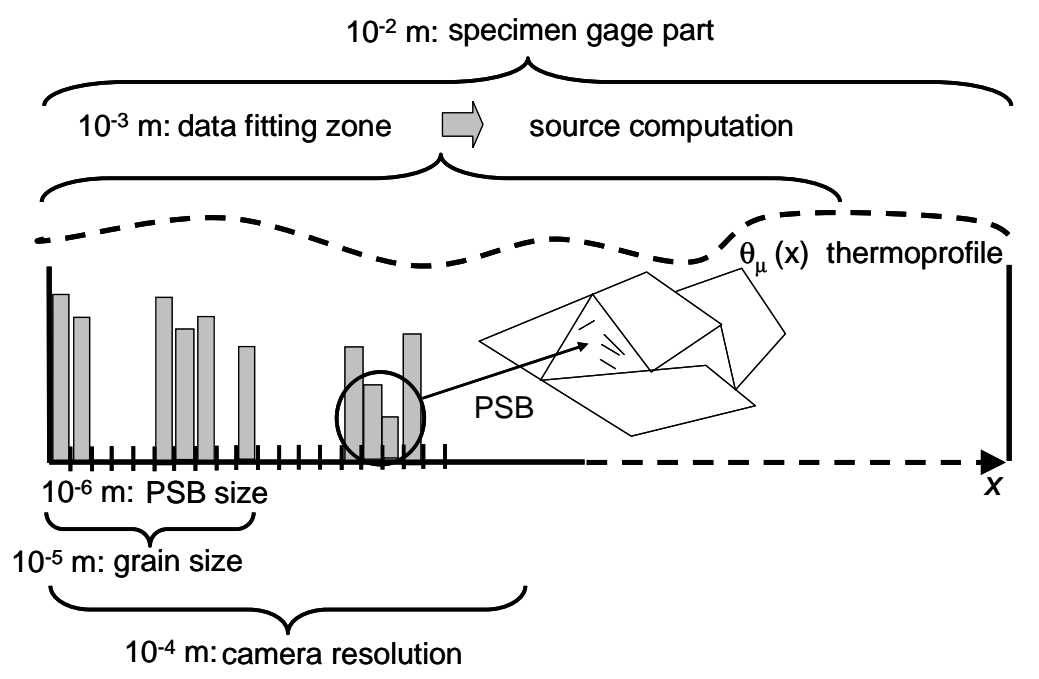

Fig. 10: Characteristic lengths associated with HCF dissipation 
Considering the $1 \mathrm{D}$ diffusion problem, it is then possible to compute the distribution of temperature at microscale $\theta_{\mu}(t, x)$ induced by a distribution of PSB. The distribution of PSB dissipation The discretization step is chosen less or equal than the PSB size. The partial differential problem can be written as:

$$
\left\{\begin{array}{l}
\rho C \frac{\partial \theta_{\mu}}{\partial t}-k \frac{\partial^{2} \theta_{\mu}}{\partial x_{\mu}^{2}}=d_{1}^{\mu}\left(x_{\mu}\right) \\
\left.\left.\frac{\partial \theta_{\mu}}{\partial x_{\mu}}\right|_{ \pm \frac{L}{2}} \mp h \theta_{\mu}\right|_{ \pm \frac{L}{2}}=0 \\
\theta_{\mu}\left(0, x_{\mu}\right)=0
\end{array}\right.
$$

where $h$ is a heat exchange coefficient. The profile of $\theta_{\mu}\left(t, x_{\mu}\right)$ is then under-sampled and averaged over a mesoscopic space step to construct a discrete thermoprofile $\theta_{\mathrm{m}}\left(t, x_{\mathrm{m}}\right)$ in accordance with those given by the IR camera. The inverse reconstruction of dissipation profile $d_{1}^{m}\left(x_{m}\right)$ can then be performed using the image processing mentioned before.
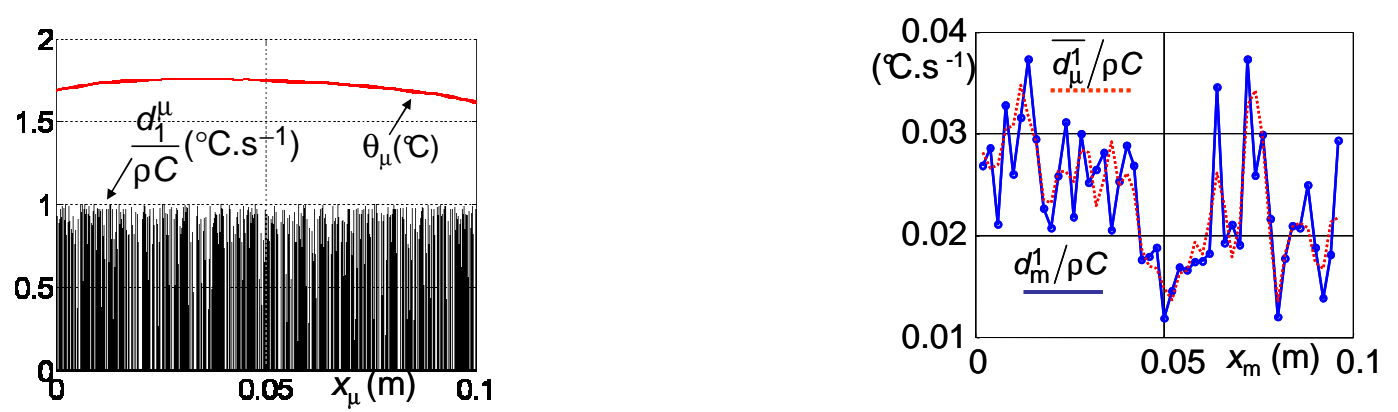

Fig. 11: Left: dissipation distribution and thermoprofile at micro-scale; right: dissipation profiles derived from temperature at mesoscale (full line) and profile averaging locally the dissipation at micro-scale (doted line).

Fig. 11 shows on the left the strong regularization effect of heat diffusion. Although the distribution of heat sources $d_{1}^{\mu}(x)$ is very heterogeneous at micro-scale, the corresponding thermoprofiles $\theta_{\mu}\left(t, x_{\mu}\right)$ are very regular. On the right, one can observe the good agreement between the averaged dissipation $\bar{d}_{1}^{\mu}$ with $d_{1}^{m}$ directly computed at mesoscale using the thermal data $\theta_{\mathrm{m}}\left(t, x_{\mathrm{m}}\right)$. Both profile show high dissipation zones whose the characteristic length is well about 1 or $2 \mathrm{~mm}$.

\section{f. deformation and dissipated energies}

In [Berthel JSA07] a complete energy balance was shown. The deformation energy on a cycle was computed by using the stress and strain data (see Eq. (11)). The stored energy $w_{s}$ icoreesponds to the internal enrgy variation (see Eq.9). An initial overview of Fig.12 shows that the mean deformation energy over a cycle $w_{\text {def }}$ (around $14.10^{-}$ ${ }^{3} \mathrm{C}$ ) is roughly made of $50 \%$ of dissipated energy and $50 \%$ of stored energy. At this level we ought to mention the work of Kaleta [14], which showed similar results at the at specimen scale. Indeed, in the case of ferritic-perlitic steel, he observed stored energy ratios associated with the stress-strain hysteresis loop varying from $37.4 \%$ for low cycle fatigue tests (LCF), and up to $67.3 \%$ for high cycle fatigue tests (HCF). In aluminium alloys, stored energy ratios were estimated from 0.1 to 0.2 in other works [29,30].

An accurate observation of Fig. 12 shows a more complicated situation induced by a heterogeneous distribution of the different energies. We already underlined the heterogeneous character of the dissipation fields induced by HCF. Here, the dissipated and stored energy vary in an initial close approximation between $4.10^{-3}{ }^{\circ} \mathrm{C}$ and $8.10^{-3}$ C.

The last point we would like to mention is related to the comparison between both energy balances. We can observe that patterns of dissipated and stored energy are very similar. We already observed this kind of feature for dissipation fields and we underlined that high dissipation zones represent potential zones where a fatigue crack may occur. Here the heterogeneous response of the material in terms of stored energy must also be mentioned. Only the time-consuming image processing prevented us from continuing the fatigue test until the 
crack inception. However, it will be interesting to also characterize these potential crack zones in terms of stored energy evolution.

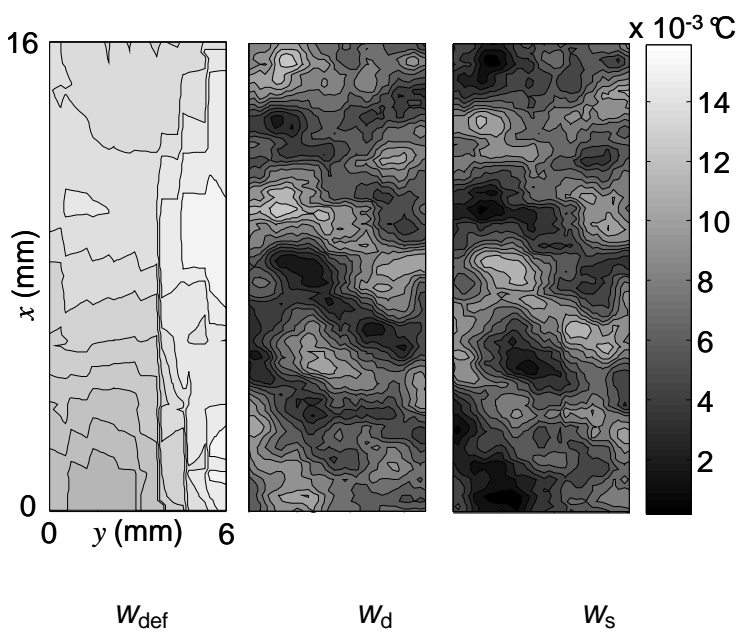

Fig. 12: Fields of the mean energy balance associated with a cycle (After Berthel et al. [JSA])

\section{Concluding comments}

In this paper, an infrared image processing method that enables estimates of deformation energy, coupling and dissipative sources accompanying HCF of steel was presented.

Applications to Dual Phase 600 steel sheets showed a linear change in the thermoelastic source amplitude as a function of the stress range and the loading frequency, in agreement with the linear thermoelasticity theory. We also observed a heterogeneous dissipation distribution from the beginning of the test. This space distribution remained time independent and did not change with the stress range and/or the loading ratio until a fatigue crack onset. Besides, the linear variation in the dissipation intensity with the loading frequency was underlined.

In terms of structure design, this method may be useful for detecting fatigue zones. The thermo-elasticity is already used to detect potential crack localisation du to stress concentrations on structures.

In terms of material analysis, obtaining the distribution of thermoelastic and dissipative sources represents precious information for anyone wishing to model the fatigue mechanisms. The coupling sources are indeed related to the constitutive state equations while the dissipation is an indicator of the fatigue kinetics and must be associated with the evolution equations. Moreover, the dissipation fields enable us to observe the fatigue mechanisms at a finer scale insofar as dissipation is mainly due to the development of persistent slip bands. Micro-analyses are currently preformed to correlate the fields of PSB density with the fields of dissipation [Lam].

\section{Reference}

